Filigrane

Écoutes psychothérapiques

\title{
Suspendre le jugement. Regard sur la psychanalyse
}

\section{Karine Roy-Déry}

Volume 23, numéro 1, printemps 2014

URI : https://id.erudit.org/iderudit/1026064ar

DOI : https://doi.org/10.7202/1026064ar

Aller au sommaire du numéro

\section{Éditeur(s)}

Revue Santé mentale au Québec

\section{ISSN}

1192-1412 (imprimé)

1911-4656 (numérique)

Découvrir la revue

\section{Citer cet article}

Roy-Déry, K. (2014). Suspendre le jugement. Regard sur la psychanalyse. Filigrane, 23(1), 101-105. https://doi.org/10.7202/1026064ar d'utilisation que vous pouvez consulter en ligne.

https://apropos.erudit.org/fr/usagers/politique-dutilisation/ 


\title{
Suspendre le jugement. Regard sur la psychanalyse
}

\author{
Karine Roy-Déry
}

Je dis souvent que se prendre pour un analyste est le commencement d'une imposture.

Jean-Bertrand Pontalis (2010)

\section{O} u'est-ce que la psychanalyse? Je n'ai jamais fait d'analyse dans le sens classique du terme, je ne me suis jamais allongée sur le divan. Je ne suis pas psychanalyste, je n'ai pas suivi le cursus de formation et ne possède donc pas la clef de la Maison. Je ne connais que quelques auteurs, principalement Winnicott, de par ma spécialisation en enfance, et encore, je ne maîtrise pas bien tous les concepts. Je m'intéresse à la pensée psychanalytique seulement depuis quelques années. Je ne suis même pas sûre de vouloir devenir psychanalyste. Disons que je suis dans l'ambivalence, autant dans mon désir de devenir analyste que dans mon désir d'analyse. Je ne suis donc ni dedans ni dehors. Je suis dans l'entre-deux, dans l'aire de jeu. "La psychothérapie se situe en ce lieu où deux aires de jeu se chevauchent, celle du patient et celle du thérapeute.» (Winnicott, 1971, 84). Je joue donc avec la pensée psychanalytique. Je me questionne par rapport à elle, comme je me questionne par rapport à moi. Les derniers dossiers de la revue Filigrane, sur l'engagement, m'ont beaucoup interpelée. J'y ai découvert des gens derrière la psychanalyse, des gens comme moi qui m’ont donné envie de parler à mon tour.

La psychanalyse me parle, m'interpelle, m'aspire, m'inspire. Je suis arrivée à la psychanalyse un peu par hasard, par la force des choses. J'ai trouvé dans la théorie psychanalytique un sens, je me suis découverte. J'y ai entendu des mots qui ont expliqué qui j'étais mais plus encore ce que je sentais. Je me suis sentie moins seule. J'y ai découvert un interlocuteur disponible, curieux de l'être humain en général, de moi en particulier. J'y ai trouvé un partenaire de questionnements, de doutes, d'incertitudes, de confusions. J'y ai appris qu'on ne peut tout comprendre, tout expliquer et que parfois même, c'est mieux ainsi. Que la vie est plus complexe que les mots qu'on pourrait y apposer. Que la vie psychique est riche, dense et que la disséquer est souvent réducteur. Que le sens est variable, et qu'il y en a plusieurs. Le regard psychanalytique 
me semble un regard ouvert, sans jugement. Il dit: «Tu n'as pas à te soucier de moi, je suis là. Je t'accompagne.»

En même temps, la Psychanalyse m'intimide. L'Institution psychanalytique m'impressionne. La majorité des psychanalystes m'insécurisent. Je sais, j'entends déjà l'interprétation tomber: «transfert négatif ». C'est une interprétation que j'accepte, car en explorant ce malaise, je réfléchis au regard de l'autre, au regard que je prête à la Psychanalyse. Je la vois comme l'Autre avec un grand $\mathrm{A}$ (comme dans les textes de Lacan). Et je m'attends à ce que cet Autre me juge. Je m'attends à un jugement. Mais, d'un autre côté, je repense à certaines discussions stériles et même violentes, à certains égards, que j'ai eues avec des "psychanalystes» et où je me suis retrouvée seule (avec mon transfert négatif).

Et je me demande pourquoi, alors qu'elle propose un regard différent, la psychanalyse est souvent perçue comme menaçante, ou jugeante. Je ne suis pas seule à avoir cette perception. Est-ce qu'un regard autre est nécessairement perçu comme menaçant? Peut-être. Mais je ne peux m'empêcher de penser que lorsque l'Autre n'est pas où il devrait être, il dérange, il menace. Il menace l'intégrité de l'organisation psychique. En étant ailleurs, le psychanalyste, se retrouve au cœur même « du trop là » (le quantitatif hallucinatoire dont parle Wilfrid Reid (Marceau, 2013)). Il nous ramène à l'intérieur même de notre rapport à l'Autre, de notre rapport à nous-mêmes à travers l'Autre.

Le propre de la situation qui a lieu dans un échange psychanalytique est d'accomplir le retour sur soi au moyen du détour par l'autre. (Green, 1998, 40-41)

Dans une section de son article « Le Contre-transfert, la symbolisation et le don d'absence», Wilfrid Reid aborde la question de l'interprétation prématurée comme un des pièges contre-transférentiels:

L'analyste est alors entrainé dans un fonctionnement psychique en miroir: sous le couvert d'interprétation, par cette rétorsion interprétative, il essaie d'évacuer la surcharge affective contre-transférentielle. Nous sommes ici confrontés au caractère contagieux du trauma psychique et bien malin qui pourra prétendre être à l'abri. (Reid, 2004, 43)

Je reprends : bien malin qui pourra prétendre être à l'abri. Je me dis qu'il doit être difficile à tolérer pour l'analyste de se retrouver constamment 
interpellé, placé dans cette position dont il se défend. Je ressens une certaine empathie pour le psychanalyste. Je me demande si toutes les techniques telles que l'interprétation, la neutralité, le silence ou même le dispositif du divan n'ont pas (aussi) pour but d'arriver à prendre une certaine distance face à ce «trop là ». Je pense que parfois, ces mêmes dispositifs qui ont pour but d'installer cette différence peuvent prendre une tout autre couleur, et représenter ce dont le psychanalyste se défend, devenant ainsi un mécanisme de défense, un retrait dans l'absence, dans une neutralité pas si bienveillante.

Nous sommes tous humains devant le trauma psychique et nul n'est à l'abri. Mais je me dis que ce qui pourrait limiter l'utilisation de la technique psychanalytique sur un mode réactionnel rétorsif, ce pourrait bien être la présence, l'attention. Je me suis beaucoup questionnée sur cette présence de l'analyste, sur le sens de cette présence, sur cet Autre qu'il incarne ou qu'il n'incarne pas. Je souligne le terme incarner que j'utilise. En effet, on peut facilement oublier l'importance d'être vivant, présent, incarné pour le client. Oublier que cette incarnation ne nous empêche nullement d'être capables de nous absenter, mais qu'elle existe. C'est ce qui m'a plu dans les textes sur l'engagement: pour s'engager, il faut exister.

Selon moi, l'enjeu fondamental du psychanalyste (ou de tout autre thérapeute) est d'arriver à se situer sur la mince ligne qui se situe entre " être là » et «ne pas être là ». Mais le problème est qu'il faut être vraiment là et vraiment pas là, à la fois! Et encore plus, si je tente de suivre le développement de la troisième topique de Wilfrid Reid (Marceau, 2013; Reid, 2008a, 2008b), être à la fois au-dedans et au-dehors.

La précarité du jeu lui vient de ce qu'il se situe toujours sur une ligne théorique entre le subjectif et l'objectivement perçu. (Winnicott, 1971, 103).

J'irais même jusqu'à proposer: pouvoir suspendre l'idée d'un dedans et d'un dehors pour lui permettre de se constituer. Cet état, cette place de l'analyste me semble difficile à expliquer, encore plus à justifier. Cette qualité de présence et d'absence se fonde sur une disposition interne de l'analyste qui peut varier dans certaines situations ou selon certains clients; comment justifier que telle parole ou tel geste soit approprié dans telle situation et pas dans telle autre? À l'époque du mode d'emploi uniformisé, une approche adaptative, variable et sensible peut facilement s'apparenter à $d u$ n'importe quoi ou du charlatanisme. 
Il est remarquable que nous ayons besoin d'experts pour nous juger, pour savoir mieux que nous ce que nous devrions faire, ou même ressentir. En cherchant à se rassurer, l'être humain semble rechercher celui qui sait mieux que lui-même et qui, en même temps le confirme dans sa position d'ignorance. Je suis convaincue que le psychanalyste, en ne se positionnant pas comme un expert, en ne donnant pas de réponses toutes faites, s'expose à la critique et que c'est la réaction à cette critique qui rendra la psychanalyse accessible. Je crois que si la psychanalyse arrive à ouvrir davantage ses doutes et ses questionnements, mais aussi ses certitudes, elle enverra le message que l'on peut être solide et douter, que les failles ne génèrent pas une angoisse insurmontable, que les opinions peuvent cohabiter et que l'Autre nous intéresse!

Seule l'implication permet de comprendre, et un sujet qui ne se sent pas compris d'un autre ne peut pas en apprendre quelque chose. On ne peut rien apprendre de quelqu'un qui ne nous comprend pas, même s'il sait très bien tout nous expliquer. (Ciccone, 2001, 97)

C'est ce que j'ai senti dans les derniers textes de Filigrane: une ouverture du psychanalyste, un désir de rencontre et l'envie de s'incarner. Je me dis que si la psychanalyse et les psychanalystes utilisent davantage cette voix, d'autres auront envie de les rejoindre sur le terrain de jeu.

Karine Roy-Déry
960, rue Jean-Talon Est, bureau 4
Montréal, Québec
H2R 1V4
k.roydery@gmail.com

\section{Références}

Ciccone, A., 2001, Enveloppe psychique et fonction contenante: modèles et pratiques, Cahiers de psychologie, no 17, 81-102.

Green, A., 1998, L'intrapsychique et l'intersubjectif en psychanalyse: Pulsion et/ou relation d'objet, Outremont, Lanctôt.

Marceau, L., 2013, Pour une métapsychologie de l'espace. Une troisième topique, Filigrane, 22 (2), 87-101.

Pontalis, J.B., 2010, Jean-Bertrand Pontalis: Pas inquiet pour la psychoanalyse, entrevue accordée à Psychologie.com, juin 2010, http://www.psychologies.com/Therapies/Psychanalyse/ Travail-psychanalytique/Interviews/Jean-Bertrand-Pontalis-Pas-inquiet-pour-lapsychanalyse. 
Reid, W., 2004, Le Contre-transfert, la symbolisation et le don d'absence, Filigrane, 13 (2), 3447.

Reid, W., 2008, Un nouveau regard sur la pulsion, le trauma et la méthode analytique. Première partie: Une théorie de la psyché, Filigrane, 17 (1), 68-94.

Reid W., 2008, Un nouveau regard sur la pulsion, le trauma et la méthode analytique. Deuxième partie: Une théorie de la méthode, Filigrane, 17 (2), 70-98.

Winnicott, D. W, 1971, Jeu et réalité, Paris, Gallimard, 2004. 